\title{
THE COMMON PRESENT IN A BLOCK UNIVERSE
}

\author{
Yuri Balashov ${ }^{1,2}$
}

\begin{abstract}
Our present experiences are strikingly different from past and future ones. Every philosophy of time must explain this difference. It has long been argued that A-theorists can do it better than B-theorists because their explanation is most natural and straightforward: present experiences appear to be special because they are special. I do not wish to dispute one aspect of this advantage. But I contend that the general perception of this debate is seriously incomplete as it tends to conflate two rather different aspects of the phenomenon behind it, the individual and the common dimensions of the present. When they are carefully distinguished and the emerging costs of the A-theories are balanced against their benefits, the advantage disappears.
\end{abstract}

\section{Introduction}

Our present experiences are strikingly different from the past and future ones. There is a sense in which the former are available to us while the latter are not. Your experience reading this paper is a case in point; some philosophers say that this experience is available to you in a way your breakfast earlier this morning is not. Or suppose I am sitting in a concert, listening to the last movement of Tchaikovsky's Sixth Symphony and having a characteristic - intensely sad and hopeless - experience associated with it, which can be usefully contrasted with the serene and uplifting experience from the previous, third movement. Again, the sad experience is available to me in a way the uplifting one is not. ${ }^{3}$

Every philosophy of time must explain these data. It has long been argued that Atheorists can do it better than B-theorists ${ }^{4}$ because their explanation is most natural and

DOI : 10.21452/LnA_serie_n_v01_book_seminario-logica-no-aviao-2013-2018_yuri-balashov_p.123-134

${ }^{1}$ Department of Philosophy, University of Georgia. Email: yuri@uga.edu.

${ }^{2}$ My thanks to Jordan Lavenver, Tom Sattig, Jeff Goodmann and the audience at the Pacific APA meetings in Seattle, Washington (April 2017) for very stimulating discussions of the issues raised in the paper.

3 To say that only the present experiences are "available" to us is just one - and by no means uncontroversial - way of making sense of the vague feeling that they are special. See Skow 2011: §5-6. The controversial part comes from insisting that 'available' means available simpliciter, not available at $t$. Alternatively, one could argue that presentness somehow shows up in the representational content of experience, or in its phenomenal character (or in both). These two ways of conceptualizing the abovenoted intuitive feeling seem to me rather implausible and inferior to the first. But taking a stand on this recently debated issue (see Skow 2011, 2015; Prosser 2016) is orthogonal to my agenda in the paper. So my use of the term 'available' in this context is rather non-committal and merely intended to give a handy label to the peculiar sense in which our present experiences are distinguished over past and future ones according to those who think they are so distinguished. Other terms can and have been used for that same purpose.

${ }^{4}$ Here and below I use the terms 'B-theory of time', 'eternalism', and 'block universe' interchangeably to denote the view that all moments of time and their contents enjoy the same metaphysical status. And I take the denial of this view to be the central feature of all 'A-theories of time.' While all A-theories - presentism, the "moving spotlight" theory, and the "growing block" theory - agree in attributing a privileged metaphysical status to the present they disagree on the nature of this privilege (see the main text immediately below). Although the issues considered in the paper affect all A-theories of time, they 
straightforward: present experiences appear to be special because they are special. For the presentist, they are special in being real or existent, as opposed to non-real or nonexistent. For the moving spotlight theorist, they are special because the spotlight of the present is shining on them. For the growing block theorist, they are special because they lie on the edge of the growing block.

These data constitute a challenge for the B-theorists who recognize no metaphysical difference among present, past, and future. Think of temporal experiences as items attached to our spacetime trajectories in the eternalist block universe spread out in four dimensions. All your experiences - eating breakfast earlier this morning, reading this paper, and many more - are equally yours, as the block universe contains a single you but many experiences, and each of them is eligible to represent your perspective on the universe. And all my temporal experiences, including the sad and the uplifting ones, are equally mine. You believe, however, that you are reading this paper rather than enjoying the breakfast. And I believe that I am having the sad experience, rather than the uplifting one. This belief in the exclusive nature of our present experiences may or may not be veridical, but it needs to be explained. B-theorists have attempted to discharge this task by combining various linguistic and metaphysical resources ranging from the semantical reduction of tense and the indexical analysis of 'present' to radically rethinking the ontology of you and $I .{ }^{5}$ While there is disagreement among the B-theoretic approaches as to how much of the above is necessary virtually everyone - the friends as well as the foes of eternalism - agree that there is some cost to be paid. ${ }^{6}$ The general perception is that B-theorists have work to do, perhaps hard work, to accommodate the apparent distinction between present and non-present temporal experiences, and that explanation does not come about so naturally as in the A-theories. As a result, the A-theories have enjoyed significant dialectical advantage on this particular score. ${ }^{7}$

I do not wish to dispute one aspect of this advantage. But I contend that the general perception of this local debate is seriously incomplete and may be distorted, as it tends to conflate two rather different aspects of the phenomenon behind it: the individual and the common dimensions of the present. When they are carefully distinguished and the emerging costs of the A-theories are balanced against their benefits the advantage disappears. Or so I argue below.

\section{Individual and Common Present}

The above examples mention our individual present experiences (IP) - your reading this paper and my listening to the last movement of Tchaikovsky's Sixth Symphony. These experiences seem exceptional to you and me, but nothing in the block universe favors them over our corresponding past or future experiences. Although this poses a prima facie problem for B-theories let us set it aside here and assume that somehow or

can be illustrated most clearly in the context of presentism, which is the main focus of my discussion; but I will make a few comments on other A-theories as well.

${ }^{5}$ For a classical B-theoretic defense of the semantical reduction of tense and the indexical analysis of 'present', see Mellor 1998. For arguments that these resources are not enough, and that explaining the exclusive nature of the present experiences requires explicit rejection of the common-sense view that persons persist by enduring, in favor of stage theory, or perdurance, see Hoy 1978; Balashov 2005, 2015, 2017; Torre 2010; and Skow 2011, 2015.

${ }^{6}$ Important exceptions include Mellor (1998: 42-45) who thinks that no cost is to be paid, that the problem of the "presence of experience" (his name for it) is easy for the B-theorist.

7 Which may, of course, be offset by the disadvantages afflicting the A-theories on other scores, from physics to truth-making. I put them aside here. 
other - perhaps by developing a convincing account of the indexical nature of $\mathrm{IP}^{8}$ or by explicitly adopting the stage view of persistence - B-theorists can handle this problem and resist the A-theoretic notion that explaining IP requires a unique and objective present. It is still true that every person has many temporally restricted perspectives on the block universe, each confined to a particular time. But let us suppose that this does not stand in the way of explaining why our 'current' individual perspectives appear so exceptional to each of us.

I submit, however, that this is only part of what needs to be explained. The other part has to do with the fact that we all seem to share a common present. As aptly put by Butterfield, "we think of ourselves as sharing a common, albeit ever-changing, now" (1984: 161), where this is explicated as follows: "two people share a now if they agree in their judgments about what is now the case, i.e. in their present-tensed judgments" (1984: 174). This characterization will be made more precise below. But to give an example, suppose you and I are at the concert together and, shaken by the experience of listening to the final movement of Tchaikovsky's Sixth Symphony, I whisper to your ear, "It's so sad!" You whisper back, "Yes, it's hopeless." Clearly, our agreement is about short stretches of our individual experiences separated by a few seconds. On a much larger scale, we also agree - in a different sense of 'agree' - that 2019 is present, that Trump's presidency is present, and so forth. Such agreement can manifest itself in many direct and indirect ways, but initially it seems to pose a separate problem for the eternalist. Even if she can explain away our beliefs in the exclusive nature of our IPs there seems to be a further problem of explaining why they are "coupled" and "coordinated" in ways that allow us to share a common present. For example, my 21:00:00 symphony-listening stage should be thus coupled with your 21:00:10 stage $^{9}$ but not with your 20:45:00 stage (which is exposed to the previous, serene and uplifting, movement). Why are they so coupled? Call this the problem of the common present $(\mathrm{CP})$.

As I understand it, my notion of CP (which will be further detailed in the next section) is very close to what Butterfield (1984) and Callender (2008) call a "common now." They both think a common now is something on which we can "agree" or "disagree," and I used this language in my initial description of $\mathrm{CP}$ above. But this use is rather special and needs a brief comment or two. First, we can disagree about a lot of things for a thousand reasons, simply because we may have different beliefs about something. We need to set all of that aside. The only agreement or disagreement of interest to us has to do with whether something is going on, or is present. Second, such agreement or disagreement is normally implicit. It manifests itself in various remarks, if we make them; in our responses to certain stimuli and prompts, if we do respond to them; and so forth. This suggests that it may be helpful to think of it as having a broadly dispositional nature. Assuming all that, how can we possibly "disagree" about the present - about what is currently going on, or about "what is now the case" (as Butterfield puts it)?

\footnotetext{
${ }^{8}$ From now on 'IP' will be interpreted more broadly to include not only individual experiences (such as seeing a green traffic light), but also individual actions (such as crossing a road) and other episodes and events, both physical and mental, involving individuals.

9 To allow for a short time lag involved in our quick communication and in perception processing. More on such time lags below. For the purpose of this discussion I use 'stage' as a neutral term not committing one to a particular view of persistence. Thus 'my 21:00:00 symphony-listening stage' is roughly synonymous with 'the 21:00:00 symphony-listening temporal stage of my life'. This is how the term was used by many philosophers (e.g. in debates about personal identity) before the recent development of the stage theory of persistence, where the term acquired a more demanding meaning.
} 
I can think of a few cases. Suppose you are watching a basketball game and listening to a commentary, and it all goes very fast. The commentator says, "Wow, isn't that great!", referring to Michael Jordan's pass to Dennis Rodman who then scores a fantastic slam dunk, which causes you to say or think, a split second later, "That is great!" Clearly, you and the commentator do not share a common present, because you react to different external events, which rapidly succeed each other. You do not disagree on the quality of Jordan's pass, but you fail to agree about its presentness.

Here is another case, on the opposite side of the spectrum. Suppose you and I communicate by email, and we both think that everything Obama does is wonderful. But I am really slow in responding to emails. You write, "Our president is wonderful, as always!" A few weeks later I get to read your email and respond (referring to Trump), "No, he is not."

These examples are not straightforward and may eventually turn on the peculiar referential behavior of indexicals and demonstratives. Why is it not straightforward to come up with examples of disagreement on the common present, on whether something is going on? I think it is because we have been conditioned by evolution to agree on it, not because it is impossible to disagree. The time lags involved in perceptual processing, the duration of the relevant external events, the way our communication goes, our reaction times, and in general the speed with which we tend to respond to various stimuli and prompts have all been shaped and selected by evolution to maximize our success. Our coherent perception of the common present ${ }^{10}$ - our implicit agreement on "what is the case" - is part of this success.

To illustrate this point, consider another case. Imagine you and I are in a jungle and there is suddenly a tiger in front of us. Suppose your relevant dispositional state, including your readiness to do certain things and avoid others, can be associated with your belief that there is a tiger in front of us, and that we should do something to save our lives. And suppose my dispositional state can be associated with my belief that there is no tiger in front of us, although there could be. In this situation, we may "disagree," in the above-noted extended sense of the term, on whether something is going on. And it may have dramatic consequences. It also explains why we rarely in fact disagree about CP in such cases. ${ }^{11}$

Characterizing these cases in terms of disagreement about a common present requires walking a fine line: we do not want an interesting concept of CP to rule out the possibility of disagreement about it; but we do want to maintain that we agree about it most of the time. Alternatively, one could say that in such cases we do not "disagree" about a common present; rather we simply lack a common present of a certain kind. And instead of saying that we "agree" on a certain CP, we could say that we share or have it. ${ }^{12}$ The problem of CP could then be put as follows: why is it that some of our IPs (e.g. my breakfast and your breakfast) can be parts of a certain CP (namely, the kind of CP that is normally associated with having meal together), but others (e.g. my breakfast and your dinner) cannot?

\footnotetext{
10 More precisely, of the many common presents; see the next section.

11 As Callender puts it, "large time lags plus fast tigers wouldn't help reproduction" (2008: 348).

12 This alternative terminology eliminates the distraction of working with the special sense of "agree" and "disagree" noted above. For stylistic reasons, and to preserve some continuity with the earlier literature, I shall occasionally use the terms agree and agreement below when talking about sharing a CP, or simply having a CP. As indicated above, agreement or disagreement in question is most often implicit, may involve broadly dispositional states, and concerns only the temporal characteristics of the relevant external events and of our IPs.
} 
When stated in these terms the problem may initially strike one as being completely trivial. And in one sense, this is entirely correct (see $\S 4-6$ below). But it is important not to lose track of where we are in our discussion at this point. We began with the B-theoretic picture of IPs scattered across the Block Universe. We then assumed that the B-theorist could explain why each of us has a particular privileged IP. Again, some think this is easy - perhaps "as easy as indexicals," to put it in a slogan. Others disagree. But we presumed that this could be done, one way or another, and that our IPs emerged from this picture as being locally privileged. So far so good. But I think we cannot stop here. Suppose my breakfast is locally privileged for me, and your dinner is locally privileged for you. Why do these IPs not belong to a common present of the relevant sort? I submit this is an interesting further question to raise, at this particular juncture.

We tend to pass over CP because it is not such an immediate - obvious and striking - datum as IP. Your current individual pleasure or pain imposes itself on you in a way that is hard to ignore. Our realization that we share or fail to share a common present is, in contrast, at a remove from immediate experience. Directing attention to it requires a shift of focus and some abstraction. And it may remain indirect even after that, lurking in some dispositional form. More importantly, it requires interaction and communication, and it involves time lags (of which more below). But despite being a bit elusive, CP is a significant fact to be accounted for. I contend that the ability to account for it is no less important in the overall cost-benefit analysis than the ability to explain IP.

Now, it might seem that the A-theories have an obvious advantage here too; for couldn't they explain IP and CP in the same uniform way by relating it to the objective ontological global present? And CP might initially look like more headache for the Btheorist; she had to work (hard) to make sense of the apparent exclusive nature of our IPs, and now she seems to be saddled with an additional problem of pairing them up in the right way.

Surprisingly (or perhaps not; see below), the situation is reversed here. The eternalist can offer a very elegant, compelling, and almost automatic explanation of CP. Indeed it is so compelling that the best strategy for the A-theorists of all stripes is simply to borrow and adapt it to their metaphysics of time. I consider this option in $\S 5$. I will argue, however, that in doing so, the A-theorists incur significant costs that undermine the unity of their position and reduce the benefits they may enjoy from IP.

\section{The Nature of the Common Present}

But the first order of business is to get clearer on the nature of CP. As already noted, our realization that we share a common present is most often implicit and indirect. We rarely ask each other questions about what is currently going on. Most of the time we simply act, communicate and interact with one another and with our environment in many different ways. CP is best thought of as a by-product of this ongoing interaction. So unlike IP, CP is not an individual experience or action, nor even a sum of such items. CP is partly constituted by IPs, but normally involves a lot more. If you and I are exposed to some external event then both the event and the relevant stretches of our IPs - those involved in our interactions with the event and with each other - become part of our common present, as we would be quick to confirm if asked. In most cases, our interaction with each other and with our environment constitutes a complex network of entangled processes including, at the very least, the external events themselves, our reactions to them, our communication with each other, as well as other related events, processes, and actions, which may be long and short, direct and mediated, active and passive, uni- and 
bidirectional, with various degrees of overlap and no well-defined boundaries. It is not possible to describe the infinite variety of such transactions. But taken together, they create a sense of a common present in us.

More precisely, each sufficiently delineated subnetwork of interrelated external events, the relevant stretches of our IPs, and our communication about them circumscribes a specific common present. The notion of $\mathrm{CP}$ so understood is causal, temporally-extended, context-relative, vague, and local. Each of these features requires a brief comment.

First, CP is causal. This is obvious from the above description.

Second, in addition to being extended in space, $\mathrm{CP}$ is extended in time. This is a consequence of its causal nature. Suppose you and I hear a roaring thunder and realize that it occurs in our common present. The CP that we thus share includes, at a minimum, certain characteristic sound wave disturbances in our vicinity, our auditory responses to them, which themselves take time, various reactions that may result from it, as well as our subsequent communication about our individual experiences. ${ }^{13}$ Besides spanning a certain region of space (say, a few thousand feet) these transactions span a finite interval of time (say, two or three seconds). Together they occupy a local spacetime region. My individual experience of the thunder, your experience of it, as well as our exchange about our experiences are all part of this CP. A worthwhile contrast here is with your experience of the lightning ten seconds earlier. The lightning is not part of the relevant common present.

On the other hand, suppose we exchange views about Trump's first presidency. Even a short fragment of our exchange may take quite some time and span a longer common present. Trump's ongoing presidency is part of this CP whose extent may range from several minutes to several days or even months (if we communicate by email or on blogs). The contrast here may be with Obama's presidency, which is no part of this CP.

This shows, third, that the temporal extent of $\mathrm{CP}$ is context-relative. What we take to be our common present may be short or long depending on the context. More examples: sharing a $\mathrm{CP}$ in the context of listening to a symphony movement requires exposure to roughly the same relatively short sequence of musical sounds. My 21:00:00 stage and your 21:00:10 stage are in this situation. Failure to be so exposed constitutes failure to share a CP. My 21:00:00 stage and your 20:45:00 pm stage are in that situation. On the other hand, living during a war sets a rather different margin for sharing a CP. Our stages separated by 15 min will normally fit in it, but our stages separated by a month may not.

The above three features of $\mathrm{CP}$ have a consequence that each of us can at any time belong to many different CPs of different extents and different degrees of overlap with each other.

Fourth, CP is typically vague in that its spatial and temporal boundaries are not precisely defined, even in a given context. Many factors contribute to this vagueness.

13 The time lags normally involved in perception and communication do not prevent us from forming a solid notion of a common present in ordinary cases of observing and touching nearby objects, or hearing sounds produced by nearby sources, or communicating with nearby persons. In most such cases the experiences we communicate to each other (and which we take to be occurring in our common present) are caused by objects and processes that change sufficiently slowly for our reports of them to be veridical and to fit properly in the margins created by the time lags of communication, observation, and so forth. The significance of such time lags in the genesis of our idea of the present has been discussed by Butterfield (1984), Stein (1991), and Callender (2008). And where the changes are very fast it is often unclear whether our observations and reports refer to the same common present. In such cases we cannot be sure that we really share a common present when communicating with each other; recall the basketball commentary example from $\S 2$. 
But as with other vague phenomena, there are clear cases of falling within a CP (in a given context) and clear cases of failure to do so. The above examples provide some such cases. Our experiences of the thunder, as well as our quick communication about them, are clearly part of the CP associated with the thunder. Your experience of the lightning is clearly not part of it. My experience of the very first sound of the thunder may be a borderline case. And all of the above are clearly part of our CP associated with Trump's presidency.

Fifth, CP is local. Its spatial extent is limited to the events that can have some, perhaps indirect, causal influence on us, and to those we can have influence on, during the finite stretch of time associated with a given CP. What is happening in the Magellanic Clouds right now (if the question even makes physical sense) is not part of our common present (i.e. no part of any of our CPs). This immediately explains why we do not care about it. In general, it is natural for us to care about what is within our "causal reach" and ignore what lies outside it. And when it comes to $\mathrm{CP}$ it is natural for us to restrict its scope to the events that belong to a causal nexus of processes directly involving or at least partially engaging our IPs. The details may be complicated and vary considerably from case to case (the contextual nature of $\mathrm{CP}$ comes into play here). But limiting the scope of $\mathrm{CP}$ to local occurrences explains why we are inclined to include in our common present a certain roaring thunder, but not a certain process, however dramatic, happening right now in Io's atmosphere. ${ }^{14}$

There is, no doubt, more to say about the nature of CP. But what has been said is enough to revisit and put into sharper focus the questions posed at the end of the previous section: Why do we share many common presents? Why does a given CP couple our IPs in the right way? What is the coupling mechanism whereby our individual, locally privileged IPs become entangled in a common present?

\section{The Common Present and Self-Selection}

First, put yourself in the shoes of an eternalist who believes in a single block universe filled, among other things, with our worldlines extending from birth to death. Various short fragments of such lines incorporate our IPs. Some of them interact with each other and with the local events, and form the notion of CP, as described above. Why do we generally "agree" on what it includes? That is to say, why do we share a certain contextrelative CP? The eternalist explanation is embarrassingly simple. Indeed, it is difficult to pose the above question without answering it. We agree on, or share, a particular CP because the agreeing (sharing) can only be done by those of our locally privileged IPs that belong to this CP.

As is clear from the description in the previous section, the common present is a causal phenomenon. Our "agreement" on a given contextually-relevant CP - our coming to share it - is just another causal process implicated in it. To agree on a given CP, we need to interact with each other and with the local events. And causal interaction of this sort naturally produces agreement thanks to a self-selection effect involved in the relevant causal transactions. Why do my 21:00:00 stage and your 21:00:10 stage agree that the music is so sad? Because the particular CP associated with it includes the short stretches of our IPs borne by our individual stages, which also do the agreeing, within the

\footnotetext{
${ }^{14}$ Such a process should not be confused with our perception of it, which may be happening right now and be part of our CP. The local nature of the common present, or "common now," has been emphasized by Stein (1991), Butterfield (1984), Gibson and Pooley (2006), and Callender (2008).
} 
relevant time margins involved in auditory processing and subsequent communication. ${ }^{15}$ In contrast, your 20:45:00 stage and my 21:00:00 stage cannot share a musical CP of the relevant sort (associated with the last - sad and hopeless - movement of the symphony), and hence cannot be constitutive of the corresponding $\mathrm{CP}$, simply because they do not belong to a common causal network of processes typically associated with fleeting musical experiences. ${ }^{16}$

The eternalist explanation of $\mathrm{CP}$ is thus very efficient: $\mathrm{CP}$ emerges as a by-product of ongoing causal interaction of the subjects of experience amongst themselves and with their environment. It originates in the self-selection of locally privileged IPs spread over the block universe. Self-selection has plenty of material to work on in the block universe! Not so in the presentist universe. Or at least not obviously so.

The presentist universe considered at a certain time includes, say, my 21:00:00 concert experience and your 21:00:00 concert experience. It does not, in addition, include your 20:45:00 concert experience. Nor does it include your 21:00:10 experience. How does a particular CP get built from the IPs available to the presentist? If the presentist were to be guided by her native ontology she should start by thinking of CP as "IP writ large." To wit, she should insist on the ontological nature of the IP-coupling mechanism: our individual IPs become part of a single CP by sharing an objective global present, and they do so in virtue of their sheer existence. If this explanatory strategy worked it would provide an elegant unified account of both IP and CP. But it doesn't work. The presentist may have a good story to tell about IP, by grounding it in the global ontological present. But the latter is at best an idle wheel in explaining CP, which has a causal and not an ontological origin.

To see it even more clearly, try this thought experiment. Posit the presentist's global ontological present but eliminate causal interaction among the subjects of experience, say, by confining them to isolated places; think of the Monte Cristo story. ${ }^{17}$ Each of them would still have a sense of a distinguished, locally privileged individual present, and the presentist could relate it to the global ontological present. ${ }^{18}$ But the subjects would not have a sense of a common present. And to the very limited extent they did - say, by gleaning incidental bits and pieces of information from prison guards, hearing occasional external sounds, catching vague glimpses of light and shadow through the window, and so forth - their limited sense of the common present (i.e. of the present they share with their immediate environment) would be causal from top to bottom. The presentist's global ontological present would be of no help in instilling it in them.

Now eliminate the objective ontological present but restore causal interaction and populate the universe with the rest of the eternalist material (i.e. the past and the future).

15 See notes 9 and 13 .

16 This does not prevent them from sharing another, more generic kind of CP associated, say, with the whole symphony.

17 Edmond Dantès, the protagonist of Alexandre Dumas's famous novel The Count of Monte Cristo, was imprisoned for many years in the Château d'If, lost track of time, and only regained it when he met a fellow prisoner, Abbé Faria.

${ }^{18}$ How? Presumably, by saying that the present experiences located in the global ontological present are all the experiences there are. The moving spotlight theorist will say that the present experiences are those in the spotlight. The growing block theorist will say that the present experiences are those on the edge of the growing block. This sense of the privileged present instant is crucial to the A-theories' respective accounts of the distinguished individual present, for it is expected to explain why subjects have a privileged sense of what they are experiencing now in the first place; it must explain the presence of their individual experiences. Different A-theories approach this explanatory task in different ways. I cannot go into details here. 
The eternalist would then have to confront the problem of the individual present and come up with some admittedly revisionary account of it. That is to say, she would need to reconcile the existence of her multiple IPs from different times with her belief in the privileged status of one of them, which she calls the present. But it is precisely the abundance of IPs from different times that also gives her a very natural way of building a common present from the multitude of individual presents by means of causal interaction and self-selection. Indeed, the eternalist does not need to "build" it. All she needs to do is watch it being self-built: the only temporal stages of you and me that can agree that they live in a common present are those that do live in a common present, since sharing a $\mathrm{CP}$ requires causal overlap. Adding the objective global present could neither add nor subtract anything from this account. This shows that the global present plays no role in it. $^{19}$

One might object that this reasoning confuses the existence of the common present with our perception or concept of it, and that the A-theorist still has a better explanation of the former. On her view, the common present exists out there, objectively, and we simply become aware of it by interacting with each other and with other objects belonging to the common present, and by making use of short-term memory. The eternalist cannot tell this kind of story about CP.

But is it a good story? What we become aware of when we form a concept of CP is not the A-theoretic present. Our concept is of the causal, temporally extended, and spatially local common present. Consider distant objects such as the Magellanic Clouds. They are every bit as real as we are, and what goes on in them right now (again, if the question makes physical sense) is part of the A-theoretic present. But is it part of our common present? No. Our concept of CP is local, while the A-theoretic ontological present is global. So if one wants to explain the origin of our CP then the eternalist's causal network of local processes, contextually bound into a particular $\mathrm{CP}$, must be the centerpiece of such explanation. It does all the heavy lifting. ${ }^{20}$

To sum up: the common present has a causal nature and is grounded in the selfselection of the individual stages of experience. The eternalist can simply take this at face value and offer a very natural explanation of $\mathrm{CP}$, which draws on the plenitude of times and of the individual temporal experiences available in a block universe.

I think this calls for a reassessment of the dialectical situation between eternalism and its rivals vis-à-vis the present. Eternalism may be revisionary when it comes to explaining the distinguished status of the locally privileged individual present experiences. But Atheorists cannot extend their native accounts of individual present experiences to the common present.

\section{Discussion}

But why should they? If the above is correct - if the common present is causal and not ontological - then what prevents the A-theorists from simply appropriating the causal

\footnotetext{
${ }^{19}$ Even if it does play a central role in explaining the subjects' privileged access to their individual present experience. This point applies to all A-theories, not just to presentism. See note 18.

${ }^{20}$ Can't the A-theorist reasonably ask whether something (or someone) in the Magellanic Clouds exists right now? If so, isn't she getting at the notion of common present? No. If she is a presentist, she is getting at the notion of existence, not of common present. If she is a moving-spotlight or a growing-block theorist, she may be getting at the notion of "lustrous existence" - a metaphor that can be developed into theories by non-presentist A-theorists. For a recent critical overview of several ways of connecting the moving spotlight of the objective present to the subjective experience of the present, see Skow 2015, which also provides useful references to earlier discussions.
} 
account of it and adapting it to their metaphysics of time? After all, eternalism has no monopoly over causation, and A-theorists of all stripes must be able to make sense of causal relations anyway. This is certainly true, and A-theorists have risen up to that challenge. But it is a well-known challenge. The cost of meeting it is especially high for presentism. Being a cross-temporal relation, causation has its natural place in the eternalist universe. The presentist, who denies the existence of more than one time, has work to do to accommodate such relations, perhaps by grounding them in presently instantiated tensed properties, or in abstracta. ${ }^{21}$ While this is no place to debate the merits and demerits of such proposals there is no doubt that they involve some cost. Causation and other cross-temporal relations are initially foreign to the presentist ontology and necessitate decidedly revisionist moves, specifically designed to recreate ersatz analogs of non-present times and their contents. ${ }^{22}$ In terms of the dialectic of the debate between the rival theories of time, the most important point here is that after such adjustments are made, it becomes clear that the main work of explaining the common present is done by the elements appropriated from the eternalist framework, where they occur naturally, not by the distinctive elements of the A-theoretic ontology.

So after the reassessment, the overall dialectical situation seems to be as follows. The B-theory may be initially unfit to deal with the locally privileged individual present experiences. To make sense of IP, the eternalist needs to do some work (or so some argue). But once this work is done, CP emerges automatically. The A-theorists, on the other hand, have an easy time with IP. But the A-theoretic notion of IP is of no use in explaining CP. To make sense of the latter, the A-theorists need to borrow the essential resources from the rival theory and pay some $\operatorname{costs}^{23}$ for adapting these resources to their respective ontologies.

In addition, by treating $\mathrm{CP}$ and IP as equally non-ontological, the eternalist position emerges as more unified in one respect. In contrast, the A-theories are, at the end of the day, committed to the view that IP and CP are entirely different in nature, the former being ontological and the latter causal.

\section{Conclusion}

Debates about the status of the present in the philosophy of time involve examples such as our current pains or pleasures and someone's present perception of a green light

21 For details of such proposals see, for example, Bigelow 1996 and Caplan and Sanson 2011.

22 The moving-spotlight and the growing-block theories may be in a better position here, as they do not deny the existence of more than one time; so there seems to be room for bona fide cross-temporal relations. I need to confine myself to the barest outline here, but things are not so simple. Consider a cross-temporal causal relation between two items $e_{1}$ and $e_{2}$ located at times $t_{1}$ and $t_{2}$ respectively. Unlike the eternalist, the moving-spotlight theorist who wants to develop a general account of causal relations cannot discharge the bulk of her explanatory task simply by pointing to these items. She needs to say that what makes them "animated" or causally effective is their presence in the moving spotlight. (This becomes particularly important in explaining the "lustrousness" of the present temporal experiences; see Skow 2011 and 2015: Ch. 11 for a useful discussion.) So she needs to say that $e_{1}$ was causally effective (as a cause) when the spotlight was shining at it; and that $e_{2}$ was (or is) causally effective (as an effect) when the spotlight was (or is) shining at it. But the spotlight cannot be shining at both times. More carefully, there is a sense in which it cannot, and this sense creates a predicament similar to the presentist's predicament. Perhaps this sense can be explained away, or shown to constitute no insurmountable problems for an account of cross-temporal causal relations. But some work needs to be done. Mutatis mutandis for the growing-block theory.

${ }^{23}$ Ranging from moderate (in the case of the moving-spotlight and growing-block theories) to significant (in the case of presentism). 
or of a sad musical passage; but also examples such as collective concert experiences, presidential elections and terms, and wars. For instance, speaking of the Second World War, an A-theorist may claim that it is objectively past, that the moving spotlight has moved away from it, or that it is already deep inside the growing block. All of that is fair game. But phenomena of the second sort may require a separate treatment. They are not anyone's individual experiences; however, they often involve such experiences. The theory needs to explain what makes a certain war present, not just for a single person, but for many people.

My discussion has taken up this task. It has assumed that the B-theorist can adequately explain why each of us has a locally privileged individual present - think of my breakfast and your dinner - and moved past this problem to a different question: what in the Block Universe disqualifies these IPs from sharing a common present of the relevant kind - the kind normally associated with having meal together? It may appear, initially, that the A-theorist already has the beginning of an answer to this question based on her account of the "local privilege." All she needs to do is simply extrapolate it to the common present. But this turns out to be a non-starter, for the common present is not assembled from IPs ontologically, rather it is self-selected from them causally. And it is the B-theorist who has the beginnings of a good account along these lines, as he embraces the whole plenitude of IPs on which self-selection can act. His ontological inventory includes my breakfast, as well as yours; but also your dinner; and a lot more. Nothing needs to be done to "assemble" a common present from them; we can simply watch it being self-assembled. This is a fascinating show, and the A-theorists need to buy into it. But they have to pay the familiar cost of recreating non-present times and their contents.

To recap, the original dialectical situation between the A- and B-theories is as follows: they agree that they both need to explain some phenomenon, IP, which is naturally at home in the A-theories, but initially foreign to the B-theory; so the B-theory faces a battle. The dialectical situation after the reassessment is different: there are two phenomena to explain, IP and CP. They are different in nature, but both need to be accounted for. IP is naturally at home in the A-theories, but foreign to the B-theory. But CP is naturally at home in the B-theory, and initially foreign to the A-theories, which need to pay some price - perhaps a familiar price - for dealing with CP.

I conclude that the general perception that the A-theories have an obvious edge over the B-theory when it comes to explaining the present is incorrect. This task has two equally important parts. The A-theories may fare better on the first, but lose to the B-theory on the second. ${ }^{24}$

24 This leaves some interesting questions unanswered. We moved too quickly past the initial problem of IP. This was done on behalf of those B-theorists who feel that they have nothing to fear from it. But the problem deserves further discussion. Knowing more about the B-theoretic account of IP would help to better understand how the theory deals with CP. More intriguingly, couldn't the potential of the B-theory to explain CP cast light on how it should deal with IP in the first place? That would be an interesting kind of "bootstrap explanation." I said earlier that it may be natural for some A-theorists to think of CP as "IP writ large"? But couldn't we instead think of IP as "CP writ small"? I cannot address these questions here. 


\section{Bibliography}

[1] Balashov, Yuri (2005), "Times of Our Lives: Negotiating the Presence of Experience," American Philosophical Quarterly 42: 295-309.

[2] Balashov, Yuri (2015), "Experiencing the Present," Epistemology and Philosophy of Science 44: 61-73.

[3] Balashov, Yuri (2017), "Time, Fission, Fusion: An Argument against the Block Universe with Endurance," Manuscrito 40 (1): 109-143.

[4] Bigelow, John (1996), "Presentism and Properties," Philosophical Perspectives 10: 35-52.

[5] Butterfield, Jeremy (1984), "Seeing the Present," Mind 93: 161-76.

[6] Callender, Craig (2008), "The Common Now," Philosophical Issues 18: 339-61.

[7] Caplan, Ben and David Sanson (2011), "Presentism and Truthmaking," Philosophy Compass 6 (3): 196-208.

[8] Gibson, Ian, and Oliver Pooley (2006), "Relativistic Persistence," Philosophical Perspectives 20: 157-198.

[9] Hoy, Ronald (1978), "Becoming and Persons," Philosophical Studies 34: 269-280.

[10] Mellor, D.H. (1998), Real Time II. London and New York: Routledge.

[11] Prosser, Simon (2016), Experiencing Time. Oxford: Oxford University Press.

[12] Skow, Bradford (2011), "Experience and the Passage of Time," Philosophical Perspectives 25: 359387.

[13] Skow, Bradford (2015), Objective Becoming. Oxford: Oxford University Press.

[14] Stein, Howard (1991), "On Relativity Theory and Openness of the Future," Philosophy of Science 58: $147-167$.

[15] Torre, Stephan (2010), "Tense, Timely Action and Self-Ascription," Philosophy and Phenomenological Research 80: 112-132. 